\title{
Serviços de editoração desenvolvidos por bibliotecas universitárias
}

Julio Santillán-Aldana

Mestre em Ciência da Informação pela Universidade de Brasília Graduado em Biblioteconomía e Ciência da Informação pela Universidade Nacional Mayor de San Marcos, Peru. Assessor e membro do International Centre for Research in Information Strategy and Development - CIEPI.Coordenador do coletivo Open Access Peru.

Suzana P. M. Mueller

Graduada em Biblioteconomia e Documentação pela Universidade Federal do Paraná. Doutora (PhD) em Information Studies pela University of Sheffield (Inglaterra, GB)e pós-doutorado na University of Illinois (III. EUA)..Professora Permanente do Programa de Pós Graduação em Ciência da Informação da Universidade de Brasília

http://dx.doi.org/10.1590/1981-5344/2644

Explora a literatura sobre os serviços de editoração oferecidos pelas bibliotecas universitárias. Mediante a revisão sistemática de literatura se tentou identificar as principais características, modalidades e experiências. Além disso, foram identificados outros aspectos importantes como a relação atual destes programas com a comunicação científica e a incidência do acesso aberto nestes serviços.

Palavras chave: Publicação científica; Comunicação científica; Serviços de editoração; serviços bibliotecários; Biblioteca universitária.

\section{The publishing service development by academic libraries}

Explores the literature on publishing services developed by academic libraries. Through a systematic review of literature. We tried to identify its main characteristics, 
methods and experiences. Complementarily other important facts were identified, such as the current relation between these programs with scientific communication and the incidence of open access in these services.

Keywords: Scientific publication; Scientific communication; Publishing services; library services; Academic library

Recebido em 02.12.2015 Aceito em 04.02.2016

\section{Introdução}

As tecnologias da informação e comunicação têm gerado mudanças significativas na comunicação científica no presente século. Esta circunstância tem motivado a ampliação da missão das bibliotecas universitárias, incluindo redefinição do seu papel e inovação dos serviços prestados, com o propósito de promover a sua adaptação às novas necessidades das instituições onde estão inseridas. Entre os novos serviços oferecidos, algumas bibliotecas estão desenvolvendo programas de editoração em linha e com isso explorando novos modelos de comunicação na academia (HAHN, 2008; CROW, 2009).

Estes serviços constituem um subcampo crescente da editoração acadêmica (SKINNER et al., 2014), que vem sendo assumindo pelas bibliotecas universitárias, estimuladas pelas crescentes demandas institucionais por plataformas de editoração digital. Como exemplo, bibliotecas universitárias estão começando a oferecer suporte à editoração de periódicos científicos, anais de congressos, relatórios, e aos sites baseados em bases em dados. A experiência acumulada ao longo do tempo no desempenho de seus serviços tradicionais somada às novas capacidades ganhas nas últimas décadas na gestão da informação capacitam as bibliotecas universitárias para esses novos serviços, nos quais prevalece a adoção de políticas baseadas na filosofia do acesso aberto à informação científica.

A tendência é que a editoração digital se torne uma competência básica dos bibliotecários e responsabilidade crescente das bibliotecas universitárias. Tal fato constitui uma mudança transcendente na filosofia de trabalho tradicional destas bibliotecas, acrescentando uma nova tarefa à sua missão tradicional de disseminadora do conhecimento: tradicionalmente as bibliotecas prestam serviços sobre informações contidas em documentos ou veículos publicados por terceiros, tais como periódicos ou livros, mas agora são chamadas a contribuir para a publicação de tais veículos. As bibliotecas universitárias, então, têm a oportunidade de se engajarem na evolução da comunicação científica como atores ativos do processo, gerando suas próprias respostas as essas 
novas demandas, e mantendo sustentabilidade econômica na disseminação mais ampla do conhecimento, de acordo com o novo paradigma da comunicação científica (WALTERS, 2012, p. 426).

Este texto buscou experiências e discussões na literatura sobre esse movimento das bibliotecas universitárias. Os resultados dessa busca são relatados nas próximas seções, assim organizadas: primeiramente, na seção 2 são apresentados conceitos e definições sobre os serviços de editoração digital oferecidos por bibliotecas universitárias, suas características e modalidades. Em seguida, a seção 3 apresenta notícias sobre experiências vivenciadas na América do Norte, Europa, Ásia e América Latina. A seção 4 discorre sobre a inserção dos serviços de editoração digital oferecidos por bibliotecas universitárias nos processos de comunicação científica. A seção 5 comenta a importância do acesso aberto nessa atividade. E finalmente, na seção 6 , conclui-se com algumas considerações sobre o tema.

\section{2 serviços de editoração oferecidos pelas bibliotecas universitárias}

Os serviços de editoração oferecidos pelas bibliotecas universitárias (SEB), denominados no contexto internacional Library Publishing Service (LPS) ou Library-Based Publishing (LBP), ou ainda Campus-Based Publishing (CBP), começaram a ser oferecidos por volta de 2007 (HAHN, 2008 , p. 13). Entre as instituições pioneiras que tem se preocupado com o tema destacam-se a organização internacional Ithaka, e a Association of Research Libraries (ARL). A organização Ithaka tem como objetivo o desenvolvimento de sistemas sustentáveis eficientes e eficazes para criação, descoberta disseminação e preservação do conhecimento, mantendo um programa dedicado ao aprimoramento de bibliotecas e comunicação científica (Library and Scholarly Communication Program) ${ }^{1}$. A ARL é uma entidade americana que reúne as maiores bibliotecas universitárias e de pesquisa dos Estados Unidos e Canadá.

Um estudo desenvolvido pela Ithaka (BROWN et al., 2007) contextualiza a situação das universidades frente à nova realidade da comunicação científica, destacando o papel de liderança que as bibliotecas poderiam assumir reunindo outras entidades na universidade, de maneira a criar uma rede para editoração acadêmica no campus. O relatório de Ithaka se complementa com o Study of Research Library Publishing (HAHN, 2008), desenvolvido pela ARL. Este estudo foi desenvolvido com a participação de oitenta bibliotecas universitárias afiliadas. Seus resultados mostraram que, na época da sua publicação, $44 \%$ das bibliotecas participantes já estavam oferecendo serviços de editoração, $21 \%$ estavam planejando desenvolver o serviço, e 36\% destas bibliotecas não estavam ativas no tema.

\footnotetext{
${ }^{1}$ Ver informações em: <http://www.sr.ithaka.org/libraries-and-scholarly-communication/>. Acesso em: 2 dez. 2015.
} 
Partindo desses trabalhos, complementados por outros citados ao longo do texto, são apresentados a seguir alguns aspectos pertinentes aos SEB.

\subsection{Definições e objetivos}

A questão da definição de termos e conceitos e a explicitação de objetivos são sempre pontos importantes no estabelecimento de novos conhecimentos e serviços. A existência ou não de consensos reflete seu estado de amadurecimento. Refletindo a novidade dos serviços, a literatura consultada traz poucas definições sobre estes serviços e seus objetivos.

Park e Shim (2011) afirmam que os SEB têm como objetivo informar e assessorar pesquisadores, autores e editores, sobre os diversos aspectos da editoração científica:

Os serviços de editoração prestados por bibliotecas são realizados para ajudar cientistas, autores e editores. Podem oferecer serviço especializado, informações no momento necessário e orientações sobre a publicação acadêmica conforme paradigma da comunicação acadêmica que muda rapidamente $^{2}$. (PARK; SHIM, 2011, p. 3, tradução nossa).

Para a Library Publishing Coalition, uma associação independente de apoio às atividades de editoração das bibliotecas universitárias, o SEB é um "conjunto de atividades dirigidas pelas bibliotecas universitárias e acadêmicas para apoiar a criação, a difusão e a curadoria das obras acadêmicas, criativas ou educativas." (LIBRARY PUBLISHING COALITION, 2013).

Contudo a definição mais difundida é aquela proposta por Hahn (2008 apud MULLINS et al., 2011, p. 11) no questionário para as bibliotecas afiliados à $A R L$ na qual o serviço de editoração da biblioteca universitária é definido como "a produção e disseminação organizada de publicações acadêmicas em qualquer formato como um serviço fornecido pela biblioteca".

É interessante destacar que esta definição, usada no questionário do Study of Research Library Publishing (HAHN, 2008), apresenta algumas explicações adicionais para estabelecer os parâmetros do SEB. Entre elas:

a)os serviços prestados pelos repositórios institucionais só podem ser considerados como SEB se a biblioteca participa de

\footnotetext{
${ }^{2}$ Library publishing services are supposed to help scholars, authors, and editors. They can provide expertise, timely notification, and advice on scholarly publishing in the rapidly changing scholarly communication paradigm (PARK; SHIM, 2011, p. 3).
} 
alguma forma no processo de produção (por exemplo, controle e validação de dados);

b)não se consideram parte do SEB os serviços de repositórios nos quais só se desenvolvem as tarefas domésticas de gerenciamento e disseminação (por exemplo, o gerenciamento de coleções de documentos); ou os serviços de digitalização (mediante scanner) ou outro modo de formato de documentos;

c)não se consideram parte do SEB os produtos e serviços desenvolvidos independentemente pelas editoras universitárias canalizados para sua difusão mediante as bibliotecas.

Outros aspectos que permitem traçar com mais detalhe a definição destes serviços são apresentados nos pontos seguintes.

\subsection{Características}

Uma das principais características do SEB, que podemos perceber na literatura, é que ele sempre está dirigido à editoração digital, ou seja, seus produtos sempre estão em versão eletrônica, portanto, produzidos em ambientes e formato digital.

Outra característica é que estes serviços desenvolvem ou participam de um processo de publicação onde os trabalhos a serem processados (manuscritos originais) não estavam antes disponíveis, são inéditos. Os SEB também participam da atribuição de certificação dos produtos gerados (conteúdos publicados), por exemplo, no controle de metadados (LPS, 2013). O SEB se diferencia das Editoras Universitárias por não ser uma unidade operativa isolada, estando normalmente integrado a outros serviços similares (HAHN, 2008, p. 21).

No Quadro 1 apresentamos uma lista de algumas características identificadas na literatura (HAHN, 2008; SKINNER et al., 2014):

Quadro 1- Características dos SEB

\begin{tabular}{c|l|l}
\hline $\mathbf{N}^{\circ}$ & \multicolumn{1}{|c|}{ Característica } & \multicolumn{1}{c}{ Detalhe } \\
\hline \hline 1 & $\begin{array}{l}\text { Estão enfocados à } \\
\text { editoração digital }\end{array}$ & $\begin{array}{l}\text { Os SEB nasceram e se desenvolvem baixo o conceito da editoração digital, ou } \\
\text { seja, na produção de conteúdo em ambientes digitais. Não tem pretensões de } \\
\text { desenvolver atividades próprias da editoração tradicional. }\end{array}$ \\
\hline 2 & $\begin{array}{l}\text { Constituem uma } \\
\text { resposta às novas } \\
\text { demandas acadêmicas }\end{array}$ & $\begin{array}{l}\text { Os estudos de casos documentados pelas diversas pesquisas sobre o tema } \\
\text { confirmam que a maioria dos programas de serviços de editoração das } \\
\text { bibliotecas universitárias se iniciaram como pequenas experiências de apoio às } \\
\text { plataformas de editoração digital ou projetos institucionais nesse sentido. }\end{array}$ \\
\hline 3 & $\begin{array}{l}\text { Tem melhor disposição } \\
\text { para a inovação }\end{array}$ & $\begin{array}{l}\text { Sendo a editoração científica um serviço adicional no seu pacote geral de } \\
\text { serviços e adotando políticas de trabalho não lucrativas (acesso aberto), os SEB } \\
\text { tem maior flexibilidade e liberdade na aplicação de novas metodologias de } \\
\text { editoração, podendo fazer inovações com maior facilidade. }\end{array}$ \\
\hline $\begin{array}{l}\text { Adota o acesso aberto } \\
\text { ecomo modelo }\end{array}$ & $\begin{array}{l}\text { Os SEB se diferenciam das editoras acadêmicas tradicionais, por seu modelo de } \\
\text { negócio baseado no acesso aberto, onde inicialmente a modalidade de receita } \\
\text { adotada é o subsidio institucional, baseado, neste caso, no orçamento da }\end{array}$ \\
\hline
\end{tabular}




\begin{tabular}{c|l|l}
\hline & & biblioteca. \\
\hline 5 & $\begin{array}{l}\text { São atores novos no } \\
\text { campo da editoração } \\
\text { acadêmica }\end{array}$ & $\begin{array}{l}\text { As bibliotecas universitárias são atores relativamente novos no campo da } \\
\text { editoração acadêmica, considerando que sua atuação se inicia baseado no } \\
\text { entorno digital nos últimos 20 anos. }\end{array}$ \\
\hline 6 & $\begin{array}{l}\text { Estão integrados a } \\
\text { outros serviços similares }\end{array}$ & $\begin{array}{l}\text { Não é uma unidade operativa isolada, sempre trabalha integrado a outros } \\
\text { serviços similares (desenvolvimento de repositórios digitais, programas de } \\
\text { digitalização, etc.) }\end{array}$ \\
\hline 7 & $\begin{array}{l}\text { Complementam e } \\
\text { fortalecem outros } \\
\text { serviços de editoração }\end{array}$ & $\begin{array}{l}\text { Os serviços de editoração das bibliotecas complementam e fazem parceria com } \\
\text { as editoras universitárias, integrando e desenvolvendo estratégias conjuntas de } \\
\text { editoração científica institucional. }\end{array}$ \\
\hline
\end{tabular}

Fonte: Adaptado de HAHN (2008) e SKINNER et al. (2014).

Um aspecto importante, que também poderia ser considerado como característica, é que o SEB constitui um serviço criado como uma resposta pragmática a uma necessidade concreta: desenvolver serviços e práticas de editoração de baixo custo, não visa lucro, dirigido exclusivamente à comunidade universitária (HAHN, 2008, p. 24).

\subsection{Modalidades}

É comum achar SEB desempenhando papel de fornecedores de serviços de hospedagem, assessores no processo de publicação de periódicos científicos, gestores de repositórios, controladores de metadados, etc. Na sua condição de serviço, pode se apresentar em três dimensões ou modalidades: assessoria, capacitação, e suporte técnico.

a)assessoria: é um serviço de orientação em editoração digital que explica, esclarece, sugere, e opina com conhecimento, sobre aspectos de produção e disseminação de conteúdos e publicações acadêmicas;

b)capacitação: é um serviço dirigido ao desenvolvimento de habilidades e capacidades para 0 uso de ferramentas e instrumentos de editoração digital (produção e disseminação de conteúdos e publicações académicas);

c)suporte técnico: são os serviços operativos e expressos de editoração digital para a resolução de problemas em sistemas, plataformas, ferramentas e instrumentos para a produção e disseminação de conteúdos e publicações acadêmicas.

Todas estas modalidades giram entorno da editoração digital baseada em acesso aberto (Veja a Figura 1 ). 
Figura 1 - Modalidades dos SEB

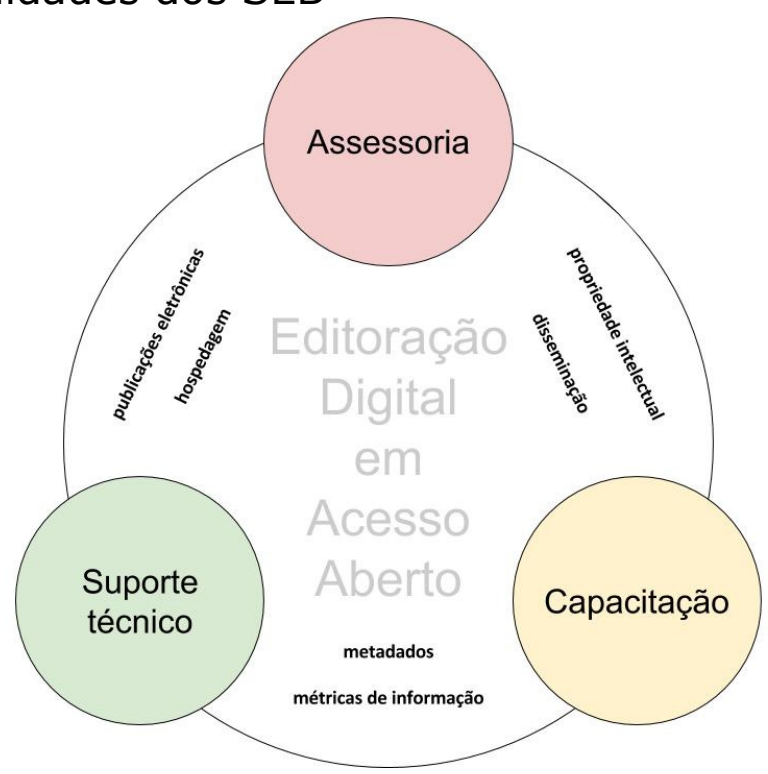

Fonte: Elaborada pelos autores (2015).

Assim são tópicos comuns de trabalho nos SEB, para cada modalidade: sistemas e plataformas de produção de publicações, metadados, disseminação de conteúdos e publicações acadêmicas, métricas de informação, propriedade intelectual, e os identificadores de conteúdo e publicações acadêmicas, entre outros.

\section{Experiências}

Com a finalidade de exemplificar de maneira mais prática os SEB, apresentamos a seguir casos documentados nas principais regiões do mundo.

\subsection{Experiências na América do Norte}

As primeiras referências sobre SEB nos países da América do Norte (Canada e Estados Unidos) se centram nas pesquisas iniciadas pela American Research Library (ARL) no ano 2007 (HAHN, 2008).

Entre janeiro de 2013 e dezembro de 2014, 61 bibliotecas universitárias, em colaboração com o Educopia Institute, fundaram a Library Publishing Coalition (LPC). O projeto surgiu das conversas entre a Purdue University, a University of North Texas, e a Virginia Tech quanto à necessidade de uma comunidade dedicada à promoção e o desenvolvimento de serviços de publicação inovadores e sustentáveis nas bibliotecas universitárias e especializadas, para que os pesquisadores possam criar e difundir conhecimento (LPC, 2013).

O número de bibliotecas com SEB nesta região vem aumentando. A última edição do Library Publishing Directory (LIPPINCOTT, 2015) apresenta um total de 105 experiências de programas de editoração de bibliotecas universitárias nos Estados Unidos e o Canadá. 


\subsection{Experiências na Europa}

Uma das principais referências na Europa Ocidental sobre SEB foi a experiência desenvolvida no Reino Unido pelo Joint Information Systems Committee - JISC (2012). Este órgão público, mediante seu grupo de trabalho em comunicação científica, apoiou a implementação em o 2011, de serviço piloto de publicação bibliotecária nas seguintes universidades:

1 University of Huddersfield - Huddersfield Open Access Publishing (HOAP);

2 University of London - SAS Open Journals;

3 University College London - EPICURE.

Outro estudo, qualitativo, sobre o tema no Reino Unido, foi desenvolvido por Lawson (2013), que identificou a presença de SEB em sete universidades (Veja Quadro 2).

\section{Quadro 2- Experiências SEB na Europa}

\begin{tabular}{c|l}
\hline \multicolumn{1}{c}{ País } & \\
\hline \hline \multirow{4}{*}{ Escócia } & Queen Margaret University \\
\cline { 2 - 2 } & University of St Andrewão \\
\cline { 2 - 2 } & University of Edinburgh \\
\cline { 2 - 2 } & University of Glasgow \\
\hline \multirow{5}{*}{ Inglaterra } & Loughborough University \\
\cline { 2 - 2 } & University of Central Lancashire \\
\cline { 2 - 2 } & University of Cumbria \\
\cline { 2 - 2 } & University of Huddersfield \\
\cline { 2 - 2 } & University of Manchester \\
\hline \hline \multirow{5}{*}{ Inglaterra } & Quadro 3- Experiências SEB na Europa (continuação) \\
\hline Irlanda & University of Northampton \\
\cline { 2 - 2 } & University of the West of England Bristol \\
\hline \multirow{2}{*}{ Suécia } & Dublin City University \\
\hline \multirow{2}{*}{ Romênia } & Stockholm University \\
\hline & Georg-August-Universität Göttingen \\
\cline { 2 - 2 } & Humboldt-Universität zu Berlin \\
\hline
\end{tabular}

Fonte: Adaptado de Library Publishing Directory (LIPPINCOTT, 2014; LIPPINCOTT, 2015) e LAWSON (2013, p. 62).

\subsection{Experiências na América Latina}

As bibliotecas das principais universidades da América Latina seguem os passos de suas homólogas nas outras regiões do mundo, e 
vêm implementando progressivamente programas e serviços de editoração.

Nos últimos anos na América Latina, e mais precisamente no Brasil, estão começando a aparecer discussões iniciais sobre o tema, explorando de forma especial o papel do bibliotecário no processo de editoração científica (MAIMONE; TÁLAMO, 2008; SANTANA, 2015). Os serviços com maior desenvolvimento na região parecem ser a gestão de repositórios institucionais e os portais de periódicos científicos gerenciados pelas bibliotecas universitárias. Nesse contexto, a demanda por profissionais da informação com capacidades ad hoc em entornos digitais vem aumentando.

Tomando como fonte os últimos rankings internacionais (CSIC, 2015; QS, 2015) das principais universidades da região, nota-se que um número significativo de universidades está desenvolvendo SEB relacionado ao conceito de Portal de Periódicos Científicos Institucionais (Veja Quadro 3).

Quadro 4 - Experiências SEB na América Latina

\begin{tabular}{|c|c|c|c|c|}
\hline País & Instituição & Área & Serviço & $\begin{array}{l}\text { Número de } \\
\text { publicações } \\
\text { gerenciadas }\end{array}$ \\
\hline Brasil & $\begin{array}{l}\text { Universidade de São } \\
\text { Paulo }\end{array}$ & $\begin{array}{l}\text { Seção de Apoio ao } \\
\text { Credenciamento de } \\
\text { Revistas USP }\end{array}$ & Portal de Revistas USP & 140 \\
\hline Chile & Universidad de Chile & $\begin{array}{l}\text { Sistema de Servicios de } \\
\text { Información y } \\
\text { Bibliotecas }\end{array}$ & $\begin{array}{l}\text { Portal de Revistas } \\
\text { Académicas }\end{array}$ & 91 \\
\hline Colômbia & $\begin{array}{l}\text { Universidad Nacional } \\
\text { de Colombia }\end{array}$ & $\begin{array}{l}\text { Dirección Nacional de } \\
\text { Bibliotecas }\end{array}$ & Portal de Revistas & 65 \\
\hline Brasil & $\begin{array}{l}\text { Universidade Federal de } \\
\text { Paraná }\end{array}$ & Sistema de Bibliotecas & $\begin{array}{l}\text { Biblioteca Digital de } \\
\text { Periódicos }\end{array}$ & 63 \\
\hline Brasil & $\begin{array}{l}\text { Universidade Federal do } \\
\text { Rio de Janeiro }\end{array}$ & $\begin{array}{l}\text { Sistema de Bibliotecas } \\
\text { e Informação }\end{array}$ & $\begin{array}{l}\text { Portal de Periódicos da } \\
\text { Universidade Federal } \\
\text { do Rio de Janeiro }\end{array}$ & 60 \\
\hline Brasil & $\begin{array}{l}\text { Universidade de } \\
\text { Brasília }\end{array}$ & Biblioteca Central & $\begin{array}{l}\text { Portal de Periódicos } \\
\text { UnB }\end{array}$ & 55 \\
\hline Brasil & $\begin{array}{l}\text { Universidade Estadual } \\
\text { de Campinas } \\
\text { (UNICAMP) } \\
\end{array}$ & $\begin{array}{l}\text { Sistema de Bibliotecas } \\
(\mathrm{SBU})\end{array}$ & $\begin{array}{l}\text { Portal de Periódicos } \\
\text { Eletrônicos Científicos } \\
\text { (PPEC) }\end{array}$ & 45 \\
\hline Brasil & $\begin{array}{l}\text { Universidade Federal de } \\
\text { Santa Catarina }\end{array}$ & Biblioteca Universitária & Portal de Periódicos & 43 \\
\hline Peru & $\begin{array}{l}\text { Pontificia Universidad } \\
\text { Católica del Perú }\end{array}$ & Sistema de Bibliotecas & $\begin{array}{l}\text { Portal de Revistas } \\
\text { PUCP }\end{array}$ & 39 \\
\hline Brasil & $\begin{array}{l}\text { Universidade Federal de } \\
\text { Goiás }\end{array}$ & $\begin{array}{l}\text { Biblioteca Central } \\
\text { Alpheu da Veiga } \\
\text { Jardim }\end{array}$ & $\begin{array}{l}\text { Portal de Periódicos da } \\
\text { UFG }\end{array}$ & 23 \\
\hline Brasil & $\begin{array}{l}\text { Universidade Estadual } \\
\text { Paulista }\end{array}$ & & $\begin{array}{l}\text { Portal de Revistas } \\
\text { Eletrônicas UNESP }\end{array}$ & 18 \\
\hline
\end{tabular}

Fonte: Elaborada pelos autores (2015).

Tem-se notado a geração de serviços complementares como: hospedagem, capacitação no sistema de gerenciamento, controle de metadados, indexação, implementação de identificadores digitais, etc. As 
experiências de trabalho das equipes dos portais de periódicos da Universidade de São Paulo (Brasil) e da Universidad de Chile (Chile) são testemunhos dos níveis de desenvolvimento que estão atingindo os SEB nesta região.

\subsection{Outras experiências}

Além do desenvolvimento dos SEB na América e na Europa, é possível encontrar experiências similares em outras regiões. Por exemplo, a última edição do Library Publishing Directory (LIPPINCOTT, 2015), dá conta de um total de cinco experiências formais de programas de publicação de bibliotecas universitárias em regiões como África e Austrália (Veja Quadro 4).

Quadro 5 - SEB em outras regiões

\begin{tabular}{c|c|l}
\hline Região & \multicolumn{1}{|c}{ País } & \multicolumn{1}{c}{ Instituição } \\
\hline \hline África & South África & Tshwane University of Technology - Pretoria Campus \\
\hline \multirow{2}{*}{ Austrália } & Australian National University \\
\cline { 3 - 3 } & Monash University \\
\cline { 2 - 3 } & Swinburne University of Technology \\
\cline { 2 - 3 } & University of Technology, Sydney \\
\hline
\end{tabular}

Fonte: Adaptado de Library Publishing Directory (LIPPINCOTT, 2015).

Informações sobre atividades de SEB na Ásia Oriental são muito limitadas, tendo sido encontradas referências sobre este serviço apenas no Japão, relatadas a seguir.

Kamada (2007) e Xia (2009) dão conta do meio da comunicação científica e seus veículos no Japão, onde a biblioteca universitária tem papel destacado. Ainda que não haja dados concretos do desenvolvimento do SEB neste contexto, há referência ao uso intensivo de repositórios institucionais e de um meio de divulgação paralelo às revistas científicas, denominado kiyo:

Esse modelo de publicação japonês, denominado kiyo, é uma variedade única de revista institucional ou departamental. Foi adotado principalmente pelas humanidades e ciências sociais, mas também tem sido usado pelas ciências, tecnologia, e medicina como uma modalidade de publicação suplementar [...] A organização dos conteúdos dos kiyo é flexível, abrangendo uma variedade ampla de tipos de publicação, de relatos de pesquisa a dados de laboratório ou notas de estudos de campo. Desta maneira, kiyo é planejado para divulgar exclusivamente os resultados de pesquisa realizadas por membros de uma unidade acadêmica, com o objetivo de trocar ideias e resultados de pesquisa e mostrar a produtividade de uma instituição de pesquisa. Por essa razão, as publicações kiyo não são oferecidas à venda e geralmente 
circulam entre pares de maneira gratuita [...] Kiyo tem uma história de quase 100 anos e atingiu o seu auge depois da Segunda Grande Guerra. Uma publicação kyio pode ser uma revista para uma disciplina acadêmica específica ou pode permitir que uma instituição em seu todo cubra várias disciplinas acadêmicas (XIA, 2009, p. 377, tradução nossa). ${ }^{3}$

Kamada (2007, p. 377) destaca com detalhes a importância do kiyo como meio da comunicação científica no âmbito das Ciências Sociais e Humanidades do Japão. Assim assinala que em 2007, o número de kiyos publicados naquele país superava os 4500 títulos, número significativo quando comparado aos 9000 títulos de revistas acadêmicas que o país publica. Informa ainda que anualmente se incorporam 150 novos títulos de kiyos. Um ponto relevante destas publicações é sua distribuição gratuita, as quais, depois de superar progressivamente os questionamentos quanto à sua metodologia de avaliação, se estão incorporando paulatinamente aos repositórios das universidades de acesso aberto mediante suas bibliotecas.

\section{Os SEB e a comunicação científica}

As bibliotecas universitárias, pela sua natureza, sempre participaram do processo da comunicação científica, facilitando inicialmente o acesso aos insumos e às fontes de informação para o desenvolvimento e pesquisa, ou seja, assumem o papel de facilitador na construção do conhecimento científico. O desenvolvimento de novas capacidades da biblioteca universitária, mediante os SEB, vem favorecendo a ampliação de sua participação no processo da comunicação científica.

Segundo Weitzel (2006, p. 88) a comunicação científica constitui um processo que envolve a construção, comunicação e uso do conhecimento científico para possibilitar a promoção de sua evolução. Neste sentido, e levando esta ideia ao plano de um fluxo, poderíamos dizer que as bibliotecas universitárias, depois de desenvolver durante muito tempo serviços de apoio ao fluxo da comunicação científica, ampliam hoje estes serviços ao nível de partícipes na construção dos veículos de divulgação do conhecimento científico.

3 [...] This Japanese publishing model, known as kiyo, is a unique variety of institutional or departmental journals. It has mainly been adopted in the humanities and social sciences but has also been used in the sciences, technology, and medicine as a supplementary publication venue [...] The organization of kiyo content is flexible, covering a wide range of publication types, from research studies to laboratory records or field notes. Therefore, kiyo is designed exclusively to publicize research outcomes by the members of an academic unit, with the goal of exchanging research ideas and results and showcasing the productivity of a research institution. For this reason, kiyo publications are not offered for sale and usually circulate for free among peers [...] Kiyo has a history nearly 100 years long and reached its peak after World War II. A kiyo publication may be a journal for a particular academic discipline or may allow an institution as a whole to cover multiple academic disciplines (XIA, 2009, p. 377). 
Por exemplo, quando Garvey e Griffith (1965) propuseram seu modelo de disseminação da informação científica há 50 anos, os limites de atuação dos serviços bibliotecários se posicionavam entre o início e a conclusão da pesquisa científica, com papel de fornecedor da informação necessária para impulsiona-la (Veja a Figura 2).

Figura 2 - Disseminação tradicional da informação científica

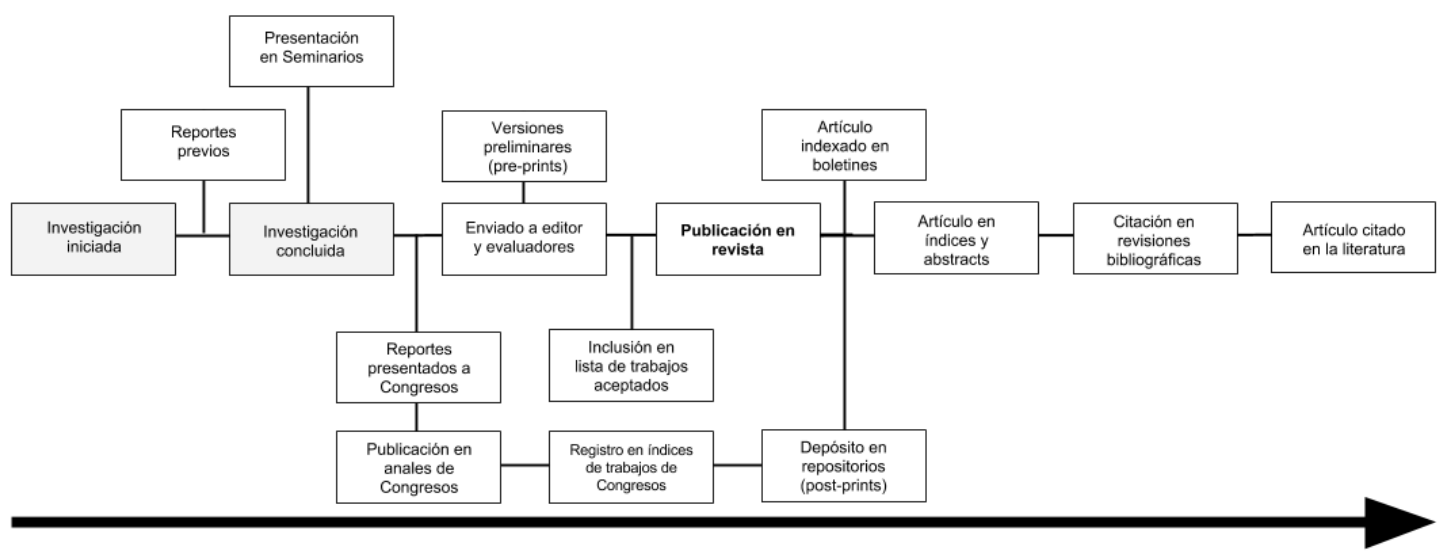

Fonte: GARVEY; GRIFFITH (1965, p. 158).

A evolução dos SEB possibilita agora uma maior amplitude na atuação da biblioteca universitária porque o fornecimento de serviços de editoração, seja para a comunicação científica formal ou informal, ampliou sua incidência no modelo da produção do conhecimento. Assim, serviços tais como 0 apoio à publicação de periódicos científicos, o acompanhamento aos sistemas de gerenciamento de congressos e o controle no depósito em repositórios institucionais, estendem o espectro dos serviços da biblioteca para novos estágios no fluxo da comunicação científica (Veja Figura 3).

Figura 3 - Disseminação atual da informação científica

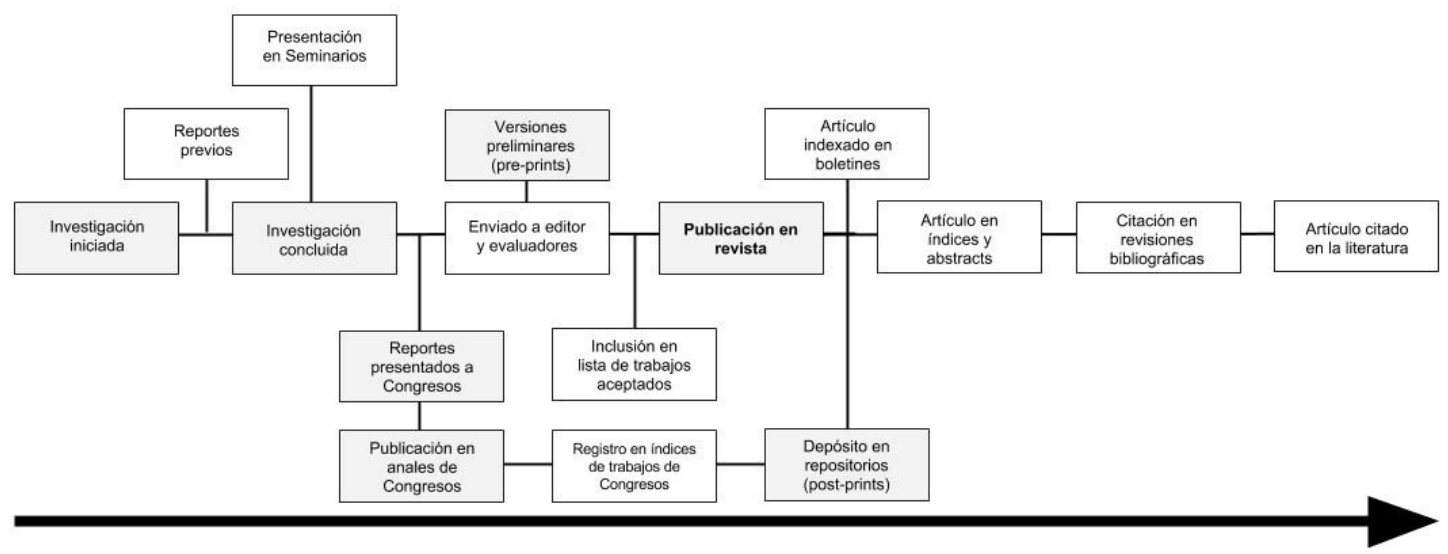

Fonte: Adaptação da versão de GARVEY; GRIFFITH (1965, p. 158).

Outro ponto complementar, que demostra a influência dos SEB na comunicação científica, é o apoio ao cumprimento de suas funções. Por exemplo, Park e Shim (2011) identificaram numa mostra de SEB etapas 
tais como registro, arquivo, certificação e serviços de alerta (awareness), os quais, na perspectiva de Roosendaal e Geurts (1997), favorecem o desenvolvimento das funções da comunicação científica. Segundo aquela análise, a maioria das bibliotecas universitárias abordam seus SEB com um olhar baseado nas funções da comunicação científica.

Assim, a função de registro se reflete nos atos de publicar, declarar a propriedade intelectual, e outorgar licenças. A função de arquivo se reflete nos serviços de digitalização e repositório. A função de certificação se reflete nos serviços de avaliação pelos pares e de apoio à pesquisa, além de assessoria sobre direitos de autor. A função de alerta (awareness) se reflete nos serviços de plataformas de intercâmbio de conhecimento, de ajuda na busca, e também nos serviços de metadados (Veja Quadro 5).

Quadro 6 - Fatores facilitadores para a comunicação científica nos SEB

\begin{tabular}{l|l}
\multicolumn{1}{c|}{ Funções da comunicação científica } & \multicolumn{1}{c}{ Fatores facilitadores identificados nos SEB (Serviços) } \\
\hline \multirow{2}{*}{ Registro } & Publicação \\
\cline { 2 - 2 } & Declaração da propriedade intelectual \\
\cline { 2 - 2 } & Concessão de licenças \\
\hline \multirow{2}{*}{ Arquivo } & Digitalização \\
\cline { 2 - 2 } & Repositório \\
\hline \multirow{2}{*}{ Certificação } & Avaliação pelos pares \\
\cline { 2 - 2 } & Apoio à pesquisa \\
\hline \multirow{2}{*}{ Serviços de alerta (awareness) } & Plataformas de intercâmbio de conhecimentos \\
\cline { 2 - 2 } & Busca e ajuda \\
\cline { 2 - 2 } & Metadados \\
\hline
\end{tabular}

Fonte: Adaptado de PARK; SHIM (2011).

A relação estabelecida no estudo de Park e Shim (2011) entre as funções da comunicação científica e os serviços oferecidos pelo SEB confirmam a contribuição das bibliotecas universitárias ao processo de comunicação científica.

\section{Incidencia do acesso aberto nos SEB}

Um último aspecto que achamos importante destacar nesta revisão de literatura sobre os SEB é o papel desempenhado pelo acesso aberto na informação científica. Como fator percussor do desenvolvimento dos serviços e editoração digital das bibliotecas universitárias, o acesso aberto tem uma importância dupla. Primeiro porque, como filosofia, permite fundamentar a importância da contribuição do SEB no contexto da comunicação científica. Segundo porque, como modelo negócio, permite entender como a biblioteca universitária se qualifica para participar como ator ativo no novo escopo da editoração científica. 
A adoção do acesso aberto levou ao desenvolvimento de instrumentos baseados no enfoque do acesso aberto, fato que constitui um elemento percussor para que as bibliotecas possam adotar com maior facilidade a função de editora digital. Referimos-nos às ferramentas para o desenvolvimento de repositórios, sistemas de edição eletrônica de periódicos científicos e de monografias, sistemas de coleta de metadados, entre outros.

\section{Conclusão}

Todos os fatos relatados nos textos citados apontam uma tendência da editoração digital para tornar-se uma competência básica das bibliotecas universitárias. A adoção deste novo papel é importante para a biblioteca universitária no cenário atual de mudanças da comunicação e da editoração científica, pois a coloca como partícipe importante da comunicação científica moderna. Nesse sentido os SEB representam uma parte da complexa da ecologia atual da comunicação científica. Os serviços de editoração desenvolvidos pelas bibliotecas universitárias têm o potencial de facilitar e ampliar o alcance da comunicação científica das instituições, exercendo influência positiva no trabalho dos diferentes atores deste processo, especialmente nas realidades dos países em desenvolvimento, onde os recursos para a pesquisa são limitados. A possibilidade da editoração digital pela biblioteca universitária se torna, então, ferramenta estratégica no desenvolvimento da ciência nesses países.

Ao longo de sua história, a biblioteca nunca deixou de se adaptar ao seu contexto, refletindo não apenas os valores sociais de cada momento histórico e sociedade, mas também absorvendo e se utilizando dos desenvolvimentos tecnológicos. A adoção da responsabilidade dos serviços de editoração pelas bibliotecas universitárias observada nos textos lidos reforça essa capacidade de adaptação e resposta, ainda que o estágio de desenvolvimento, em cada região do planeta, mostre diferenças marcantes. No entanto, esse parece ser um processo em pleno desenvolvimento, e o cenário poderá se mostrar bastante diferente nos próximos anos.

\section{Referencias}

BROWN, L. et al. University Publishing in a Digital Age. The Journal of Electronic Publishing, v. 10, n. 3, 2007. Disponivel em: $<$ http://dx.doi.org/10.3998/3336451.0010.301 >. Acesso em: 2 dez. 2015.

CROW, R. Campus-based publishing partnerships: a guide to critical issues. Washington, D.C.: SPARC, 2009.

CSIC. Ranking Mundial de Universidades en la Web. 2015. Disponivel em: <http://www.webometrics.info>. Acesso em: 15 out. 2015. 
GARVEY, W.; GRIFFITH, B. Scientific communication: the dissemination system in psychology and a theoretical framework for planning innovations. American Psychologist, v. 20, n. 2, p. 157-164, 1965.

HAHN, K. Research library publishing services: new options for university publishing: New options for university publishing. Washington, DC: Association of Research Libraries, 2008. 40 p. Disponivel em: $<$ http://www.arl.org/storage/documents/publications/research-librarypublishing-services-mar08.pdf >. Acesso em: 2 dez. 2015.

JOINT INFORMATION SYSTEMS COMMITTEE. Scholarly communications. JISC, 2012. Disponivel em: <https://www.jisc.ac.uk/rd/projects/scholarlycommunications>. Acesso em: 21 ago. 2015.

KAMADA, H. Kiyo Journals and scholarly communication in Japan. Libraries and the Academy, v. 7, n. 3, p. 375-383, 2007.

LAWSON, S. Library publishing services: an investigation into open access publishing in academic libraries. 2013. $68 \mathrm{f}$. Dissertation (MA Information Studies) - School of Computing, Engineering and Mathematics, University of Brighton, Brighton, 2013. Disponivel em: $<$ http://eprints.rclis.org/25156/1/Lawson2013 dissertation_LibraryPublish ing.pdf >. Acesso em: 2 dez. 2015.

LIPPINCOTT, S. (Ed.). Library Publishing Directory 2016. Atlanta: Library Publishing Coalition, 2015.

LIPPINCOTT, S. (Ed.). Library Publishing Directory 2015. Atlanta: Library Publishing Coalition, 2014.

LIBRARY PUBLISHING COALITION. What is library publishing? Atlanta, 2013. Disponivel em: <http://www.librarypublishing.org/about-us $>$. Acesso em: 2 dez. 2015.

LIBRARY PUBLISHING SERVICE (LPS). What is library publishing? Library Publishing Coalition, 2013. Disponivel em: <http://librarypublishing.org/about-us>. Acesso em: 21 ago. 2015.

MAIMONE, G.; TÁLAMO, M. D. F. A atuação do profissional da informação no processo de editoração de periódicos científicos. Revista ACB: Biblioteconomia em Santa Catarina, Santa Catarina, v. 3, n. 2, p. 301321, 2008.

MULLINS, J. et al. Library Publishing Services: strategies for success research. Report Version 1.0. Washington: SPARC, 1 nov. 2011. Disponível em: $<$ http://docs.lib.purdue.edu/cgi/viewcontent.cgi?article $=1166 \&$ context $=$ lib research>. Acesso em: 2 dez. 2015.

PARK, J.-H.; SHIM, J. Exploring how library publishing services facilitate scholarly communication. Journal of Scholarly Publishing, Toronto, v. 43, n. 1, p. 76-89, 2011. 
QS. University Rankings. 2015. Disponivel em: <http://www.topuniversities.com/university-rankings>. Acesso em: 15 out. 2015.

ROOSENDAAL, H.; GEURTS, P. Forces and functions in scientific communication: an analysis of their interplay. Cooperative Research Information Systems in Physics. Oldenburg: [s.n.], 1997.

SANTANA, S. O bibliotecário e a editoração de periódicos científicos: fazeres e competências. São Paulo: Escola de Comuicações e Artes; Universidade de São Paulo, 2015.

SKINNER, K. et al. Library-as-publisher: capacity building for the library publishing subfield. The Journal of Electronic Publishing, Ann Arbor, Michigan, v. 17, n. 2, 2014. Disponivel em: < http://dx.doi.org/10.3998/3336451.0017.207>. Acesso em: 2 dez. 2015.

WALTERS, T. The future role of publishing services in university libraries. Libraries and the Academy, v. 12, n. 4, p. 425-454, 2012.

WEITZEL, S. da R. Fluxo da informação científica. In: POBLACIÓN, D. A.; WITTER, G. P.; SILVA, J. F. M. Comunicação \& produção científica: contexto, indicadores e avaliação. São Paulo: Angellara, 2006. cap. 3, p. 82-114.

XIA, J. Library publishing as a new model of scholarly communication. Journal of Scholarly Publishing, v. 40, n. 4, p. 370-383, 2009. 